\title{
A LAYERED FRAMEWORK FOR CONNECTING CLIENT OBJECTIVES AND RESOURCE CAPABILITIES
}

\author{
ASIT DAN \\ IBM T.J. Watson Research Center, 19 Skyline Drive, Hawthorne, NY 10532, US, asit@us.ibm.com \\ CATALIN L. DUMITRESCU \\ Delft University of Technology, Mekelweg 4,Delft, 2628 CD Delft, the Netherlands, c.dumitrescu@ewi.tudelft.nl \\ KAVITHA RANGANATHAN \\ IBM T.J. Watson Research Center, 19 Skyline Drive, Hawthorne, NY 10532, US, kavithar@us.ibm.com \\ MATEI RIPEANU \\ The University of British Columbia, 2332 Main Mall, Vancouver, BCV6T 1Z4, Canada, matei@ece.ubc.ca
}

\begin{abstract}
In large-scale, distributed systems such as Grids, an agreement between a client and a service provider specifies service level objectives both as expressions of client requirements and as provider assurances. From an application perspective, these objectives should be expressed in a high-level, service or application-specific manner rather than requiring clients to detail the necessary resources. Resource providers on the other hand, expect low-level, resource-specific performance criteria that are uniform across applications and can easily be interpreted and provisioned.

This paper presents a framework for service management that addresses this gap between high-level specification of client performance objectives and existing resource management infrastructures. The paper identifies three levels of abstraction for resource requirements a service provider needs to manage, namely: detailed specification of raw resources, virtualization of heterogeneous resources as abstract resources, and performance objectives at an application level. The paper also identifies three key functions for managing service-level agreements, namely: translation of resource requirements across abstraction layers, arbitration in allocating resources to client requests, and aggregation and allocation of resources from multiple lower-level resource managers. One or more of these key functions may be present at each abstraction layer of a service-level manager. Thus, layering and the composition of these functions across abstraction layers enables modeling of a wide array of management scenarios. The framework we present uses service metadata and/or service performance models to map client requirements to resource capabilities, uses business value associated with objectives to arbitrate between competing requests, and allocates resources based on previously negotiated agreements. We instantiate this framework for three different scenarios and explain how the architectural principles we introduce are used in the real-word.
\end{abstract}

\section{Introduction}

In large-scale, service-oriented systems like today's Grids [1], a client may establish a service level agreement (SLA) [2-4] with a service provider prior to the actual invocation of the service. Such an agreement provides assurance to the client on the availability of requested 
resources and/or on service performance. The agreement may include one or more guarantee terms on service level objectives, (e.g., average service response time less than one second). A guarantee term may also include the importance of meeting the objective (e.g., expressed as a penalty for not meeting the objective) and additional qualifying conditions (e.g., duration of the contract) [5,6]. The presence of service level objectives (SLOs) enables flexible allocation of resources at the service provider. First, resources can be allocated dynamically to meet the SLO only when a client invokes the service, leading to higher resource utilization through resource sharing across client requests. Second, when a peak in offered load causes a resource conflict the provider can make informed resource allocation decisions and maximize the business value associated with the requests it chooses to serve.

However, finding the appropriate level of abstraction for SLOs is non-trivial. For ease of use, clients would like to express their objectives in terms that are meaningful to them, such as response time, or even as abstract expressions of processing requirements, e.g., required MIPS. With an abstraction at this level clients do not have to specify resource details, such as requirements on cache or processor characteristics. To facilitate this abstraction, however, a resource provider needs to present its capabilities in abstract terms. Furthermore, the provider needs to be able to interpret offered SLOs and determine the detailed resource configuration necessary to meet them.

Managing client objectives can also require aggregating resources from multiple resource providers. To avoid the overhead and the latency of resource acquisition, an intermediary may acquire resources for longer intervals thus enabling reuse across client requests. Figure 1 illustrates this scenario: the intermediary acts as a resource aggregator on clients' behalf and establishes SLAs with each resource provider.

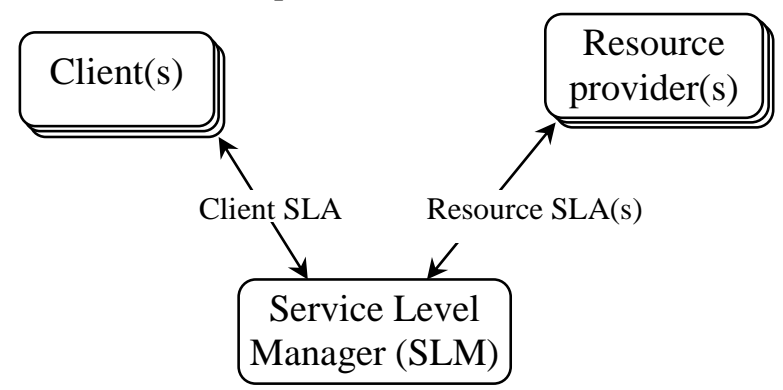

Figure 1: The Service Level Manager (SLM) bridges the gap between client and resource provider abstraction levels.

The major challenge is to provide the right level of abstraction: SLO parameters (e.g., granularity, duration) that can be met by the provider while requiring low resource management overheads, (i.e., help avoiding resource reconfiguration) and promote high resource utilization.

In this paper we introduce a framework for bridging the gap between the high-level specification of client objectives and the detailed specification of resource capabilities managed by a resource manager. This framework, that we refer to as the Service Level 
Manager (SLM), performs three essential functions. Firstly, the SLM uses detailed service performance modeling to map client objectives to resource requirements. Secondly, it acquires and aggregates resources, possibly from multiple resource managers, and maintains bindings to these resources. Finally, the SLM keeps track of client established SLAs and arbitrates dynamic allocation of resources across multiple client requests. We design the SLM as a service oriented architecture [7] for its ease to aggregate and coordinate multiple, service-based components (based on Grid services for example) and present our experience with three application prototypes instantiating this architecture for application specific scenarios.

The paper is organized as follows. Section 2 uses a concrete example to illustrate how client objectives can be expressed at various levels of abstraction. We show that detailed specification of resource requirements is both cumbersome for clients and results in resource underutilization. Raising the abstraction level on the other hand introduces challenges in expressing and managing client objectives. Section 3 examines the architectural requirements for supporting the three key SLM functions, and the composition of these functions in a layered architecture. Section 4 presents our prototyping experience while Section 5 explains how SLM functions and existing SLM systems can be composed to address new scenarios. We summarize and conclude in Section 6.

\section{Client and Resource Provider Objectives}

We first describe a real-world scenario to better motivate the need to bridge the gap between client objectives and resource provider capabilities. We refer to this scenario throughout the rest of the paper. This section then describes possible abstraction levels at which clients and resource provider can specify their performance objectives. Our description is not intended to be exhaustive or prescriptive, we are rather interested in exploring the space of performance objectives and resource requirements that need to be expressed using SLAs.

\subsection{Motivating Scenario}

Consider this sample scenario in a financial company [8]:

An investment-bank, active in multiple financial markets, owns computational resources in each country where it operates. These resources are primarily used as trading screens. Since traders are active only during the day, the bank decides to run a portfolio risk evaluation, a compute and data intensive application, on these resources at nighttime. One strong requirement is that results be available before 7:00 am, the start of the trading day, in each market.

The application consists of two stages. First it solves a large system of linear equations. This is a parallel, tightly-coupled, compute and network intensive computation. The result, together with new input, is used in the second stage organized as a master-worker: workers compute the risk profile for a set of trades, send results back to the master, and receive a new set of trades to price. The master assembles all information received into the final result.

In each market, at the end of the trading day, a new computation is started with a 7:00 am next day deadline expressed as an SLA. This application-level goal is translated into lower level SLAs for each stage of the application, and is either matched with existing services or with new services created on 
demand. If available resources are scarce these services are instantiated at a utility provider with which the bank has negotiated outsourcing agreements. If SLA violations are reported, a repair mechanism is invoked: it reevaluates the feasibility of producing the final result before the deadline and might acquire additional resources to speed-up the computation (through the same SLA mechanism).

A related scenario is suggested by Leff et al. [9]: at peak loads, Internet service requests with a given average response time goal are served using resource preempted from the long-running application.

A number of requirements emerge from these scenarios. Client applications need to express performance objectives at various levels: in our example the portfolio evaluation deadline is a performance objective that is in turn translated into deadline and/or throughput requirements for each application stage. Resource providers, on the other hand, express their offers and negotiate contracts in resource specific terms. A separate component is needed to bring clients and resource providers together, to monitor progress, and to take action if progress does not meet expectations. Additionally, this runtime framework needs to match client and resource provider SLAs, to dynamically allocate resources either internally or externally (e.g., from utility providers), and to dispatch requests according to the SLAs that have been created.

\subsection{Resource Provider Objectives}

Resource providers handle low-level, resource-specific performance objectives that (ideally) are application independent and can be easily interpreted and provisioned for. We envisage three levels, at which resources or groups of resources can be abstracted: firstly, at the raw resource level, resources are identified individually, secondly at the abstract resource level, qualitative and quantitative performance objectives are specified characterizing individual or groups of physical resources, finally, at the more complex service level performance objectives are tied to the execution of a specific service.

\subsubsection{Raw Resource Level Objectives}

At the lowest level, service level objectives (SLOs) include detailed resource configuration information: they name or individually describe the physical resources requested (e.g. "I need 16 processors on cluster X"). A slightly more involved step is to describe objectives in terms of predefined resource attributes. Note that this implies that the client has prior knowledge about the characteristics of the resource requested.

There are two important drawbacks for presenting SLA at this level of abstraction. First, it imposes a burdensome level of detail on the clients that need to fully specify SLAs when acquiring resources from providers. Second, fixed allocation reduces resource management flexibility: it does not allow for resource sharing across client requests and may result in inefficient resource utilization. While this level of detail might be required in certain cases, we are interested in expressing abstract, higher-level objectives that can be translated into lower-level resource requests by the run-time infrastructure. 
Related work: A number of schedulers, resource management frameworks and resource specification languages routinely present resources at this level of abstraction: e.g, Loadleveler [10], the Maui scheduler [11], LSF [12], the Globus Toolkit's Grid Resource Management Service (GRAM) [13] and its associated Resource Specification Language (RSL), or the Job Submission Definition Language (JSDL) [14].

\subsubsection{Abstract Resource Level Objectives}

To quickly convey intuition we start with an example: the resource provider objective in the example above can be restated as "I need aggregate compute power of 3.2 GFLOPS and aggregate memory of $8 \mathrm{~GB}$. The communication pattern of my application is a $2 \mathrm{D}$ mesh and the communication/computation ratio is $1.2 \mathrm{Mbps} / \mathrm{MFLOP}$ ".

A resource provider will accept these higher-level SLOs and translate them to raw resource requests. Obviously this provides more flexibility: the resource provider can now allocate resources appropriately, and, in effect, take over the task of finding appropriate resources from a client that does not have - or does not care about - detailed resource information.

Some of the performance related objectives that can be used: CPU related metrics (e.g., aggregates of MIPS or MFLOPS), memory related metrics (e.g., aggregated memory size and CPU to memory bandwidth), storage related metrics (e.g., total space to be used, read/write aggregate throughput), network related metrics (e.g., aggregate generated traffic, traffic generated per floating point operation). SLOs using these metrics can be used to express either 'strong' deterministic objectives (e.g. the system will always deliver more than $\mathrm{N}$ units of the metric) or as statistical guarantees (e.g., averages over some time period). Specifying the time period over which averages are computed and variability are possible refinements.

In addition to performance related objectives, qualitative objectives are generally necessary. We list a few examples:

- Heterogeneity levels. While a large number of parallel applications can efficiently cope with heterogeneity, some applications require homogeneous resources.

- Hardware/software configuration. For example, some applications might require machines that implement a specific floating point operation standard.

- Reliability guarantees might be important for long-running client applications

- For some applications, application topology information is crucial to efficiently select and configure resources.

\subsubsection{Application Level Objectives}

In addition to providing raw resources where clients instantiate their own services, the resource provider might move up in the value chain and itself provide services. A suitable example are scientific libraries (e.g.: BLAS [15], SCALAPACK [16]) which can form the basis of a set of linear algebra services offered by a provider [17]. 
Below, we suggest a few performance objectives at this level. As in the previous sections, we only present single objectives; nothing prevents clients to negotiate SLAs that include multiple objectives.

- Time related client objectives cover a large part of the usage space. Per-request service time objectives can either specify a guaranteed service time upper bound or averages over longer periods. Similarly, throughput objectives specify the number of requests served during a time interval of specified duration.

- For more complex services, resource providers may still express goals in terms of resource consumption (CPU, generated traffic) delivered to an application. The significant advantage is that, for a large class of applications, the service topology and generated network traffic are abstracted away and the performance of the service can be expressed using only one of the metrics described earlier.

- Additionally specific, quality of solution objectives (e.g., the solution to an optimization problem approximates the best solution with certain accuracy) or reliability objectives (e.g., service uptime, or data availability.) can be included. Client objectives may also include assurances on resource availability at a future time, i.e., advance reservations. Providers may express confidence level on the availability of resources at a future time.

Note that, from a resource provider perspective, accepting commitments at this level is risky unless the application/service behavior is well understood and faithfully modeled. There are multiple challenges: firstly, applications with nonlinear performance or resource consumptions are not uncommon, and, for some classes of services it is impossible to accurately estimate beforehand the work required to serve each particular request. As a result significant overprovisioning might be required. Secondly, managing objectives at this level sometimes implies aggregates over multiple resource, resource types, or resource providers.

Related work: Some runtime environments for structured parallel programming (e.g., ASSIST [18], GrADS [19], HOCs [20], Edilali et al. [21]), aim to reduce or hide the complexity of resources presented to users. These environments however have different foci: firstly they target relatively homogeneous resources under a single administrative authority; secondly they do not aim to enable composition of dynamically created services by standardizing their interfaces and a common language to express performance objectives.

\section{Key Service Level Manager Functions: Connecting Client and Resource Provider Objectives}

This section presents the key functions needed to bridge the abstraction gap between high-level client requests objectives and lowelevel resource requirements, examines the architectural requirements for supporting these functions, and their composition in a flexible, layered architecture. 


\subsection{Resource Management and the SLM}

We argue that the Service Level Manager (SLM) needs to fulfill three main functions:

- Resource and environment identification. Here high-level client performance objectives are translated into detailed resource requirements and identification of the environment. To achieve this goal the SLM needs two types of information: accurate modeling of service/application performance and timely information on available resources. The SLM translates high-level performance objectives into raw resource requirements that are allocated by resource managers at the resource provider.

- Matching and Arbitration. The SLM matches the available resources with detailed resource requirements and arbitrates across competing requests to produce a resource allocation that maximizes the 'utility' delivered to client applications.

- Aggregation and Allocation of Resources. Actual physical resources are allocated and/or the environment is configured prior to application execution. When multiple resources are involved in serving a single request or in instantiating one service, the SLM is also the coordinator in acquiring and managing these resources. Resources involved might belong to multiple administrative domains and/or might be of different types (e.g., for the transfer of large data-sets between two organizations computational capacities and storage need to be reserved at each end as well as network capacity between endpoints).

To avoid developing new custom code for each new service, these SLM functions are developed in a general manner (referred to as "middleware"), and reusable across a wide range of services [3].

\subsubsection{Resource and Environment Identification}

There are multiple possible approaches to map a high level objective to a detailed resource configuration. The choice of a specific approach depends on the application objectives being modeled. Prior to creation of a SLA, a provider specifies SLA templates that describe the parameters that can be customized by clients $[2,3]$. Hence, the most straightforward approach is to use a table for mapping a combination of customizable SLA parameters to a set of resource configuration parameters. Such tables can be built using analysis, heuristics, or application specific benchmarking for specific resource configurations. Clearly, this approach can work only if a small number of parameters are to be changed across different SLAs with clients, and/or a small set of services are to be modeled by the reusable SLM code.

To handle a larger set of SLAs, and hence, derived resource configurations, mapping requires use of customized application models. Building such a model, however, is not straightforward and requires domain knowledge [22-24]. In Section 4.2. we present our experience with a service-based prototype embedding a detailed performance model for a high-performance scientific-computing application, the Cactus framework. Other possible approaches include extrapolation from a known set of configurations as advocated in [25] or automatically extracting application performance models $[19,26]$. In addition, for particular 
classes of applications (e.g., divide-and-conquer, parameter sweeps) is it possible to monitor the application progress and dynamically size the resource pool according to the progress rate observed $[27,28]$.

\subsubsection{Matching and Arbitration}

Once the requirements for a specific SLA are identified, the next step is matching these requirements onto available resources [29-32]: that is, discovering sets of candidate resource that meet these requirements. Information on available resources characteristics, on their status, and on other existing SLAs, need to be taken into account. The result of the matching process is a set of pre-allocated resources that meet SLA objectives. When multiple concurrent requests are present and sufficient resources to meet the SLAs are not available, then allocations need to be prioritized based on the business value or utility associated with meeting a service objective [2]. This process is referred to as arbitration or conflict resolution.

Traditionally, resource management systems have focused on optimizing system-centric performance metrics such as mean computation time, or average system utilization [13, 33, 34]. These resource management systems use combinations of priorities and fair sharing to express resource allocation policies [11,35, 36]. These mechanisms, however, do not express the value clients attach to fulfilled service requests or the value resource providers associate

with their assets. To take perceiver utility into account, the SLA based management introduces two value related metrics: (1) a utility value associated by the client to a served request, and (2) a penalty function for not meeting the specified performance objectives. The penalty function can be as simple as saying that no value is delivered if performance objectives are not met, to more sophisticated functions: e.g. a decreasing value function over time for time related objectives [6, 37, 38]. Aman et al. [39] describe a workload management system that uses adaptive algorithms for managing multiple client objectives. The algorithm dynamically learns the resource requirements for meeting an objective, and adjusts various types of systems resources allocated to an application (e.g., MIPS, buffer size, etc.). Dan et al. [40] use SLA based web-services management and request prioritization to manage average response time goal.

\subsubsection{Allocation and Binding}

Once a resource pre-allocation decision has been taken, making this decision effective may be as simple as marking a specific node as allocated, and starting a job or application in the allocated node. However, this may also mean provisioning or reconfiguring a server for use by the selected application. In a shared resource environment, this physical allocation process is continual: dynamic adjustments of resource allocations are generated, either by a change in resource allocation policies, in execution priorities, or a reconfiguration of the execution environment by starting, stopping or migrating the application to new server nodes [38, 39]. If 
the resources used by an application are managed by multiple independent managers (e.g., multiple independent clusters, decomposed into multiple application tiers, decomposed into storage, database and application server, etc.), then the allocation or reconfiguration decision is conveyed to all the resource managers [38]. Here, the application-level SLM may have lower-level SLAs with the individual resource managers.

\subsection{Composition of SLM functions}

One can view the SLM as a vertically integrated component providing these three functionalities we have just presented. However, we believe that a layered view that matches the virtualization levels described in Section 2 is a better solution for architecting the SLM. Thus, we loosely separate SLM in three functional layers that match the level of abstraction for resource management at resource providers presented in Section 2.2:

- Layer 1: Service-level resource management. At this layer application/service specific performance and structural models are used to translate high-level performance objectives to abstract resource requirements and to infer the categories of resources needed.

- Layer 2: Abstract resource management. This layer uses information published by resource providers (and obtained through monitoring mechanisms) to translate abstract performance objectives to requests for concrete set of resources.

- Layer 3: Raw resource management. We include this layer here only for completeness as, in fact, this is the layer where most existing resource managers (e.g., cluster schedulers $[10,11,34]$ or network reservation mechanisms [41]) already operate to perform raw resource acquisition.

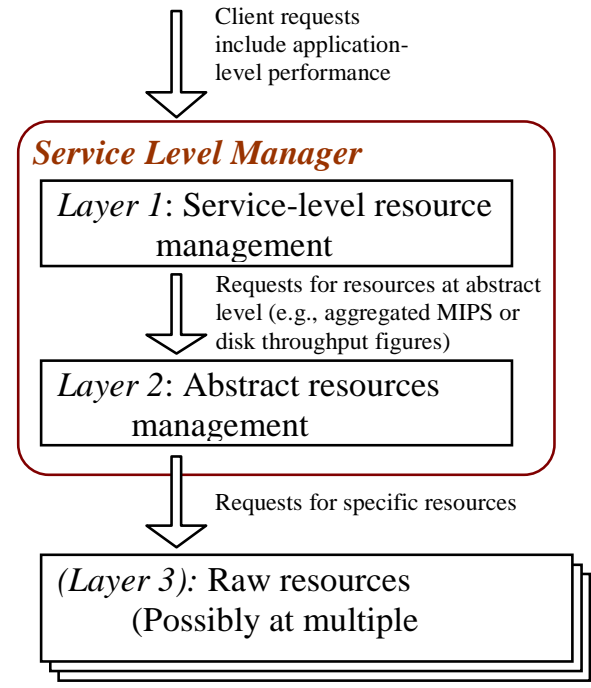

Figure 2: Service Level Manager (SLM) layer decomposition 


\subsubsection{SLM Layer 1: Service-Level Resource Management}

This SLM layer translates application-level performance objectives resource requirements at the abstract resource level. Additionally, this layer interfaces with service factories that encapsulate all service/application specific details: at a minimum, factories incorporate service implementation requirement details. This includes not just the information needed to map the service components to resources, and/or information needed to start and configure the service, but also a performance model to quantify the amount of resources required.

A service instantiation request includes service performance objectives and notification conditions. Before accepting the request, this layer collaborates with the service factory to instantiate the service. The layer provides a set of reusable functions [3] that are customized with information specific to a service factory (e.g., a database table entry for each service factory listing required resources of different types). The handle of the newly created service instance is returned to the client to submit actual request(s) or to subscribe to notification events. All subsequent invocations of the service are transparent with regard to actual low-level resource management or resource aggregation (co-scheduling and co-reservation) $[25,42]$. The layer in requesting resources from the next layer passes business value associated with this request, in case arbitration is needed at the next layer.

\subsubsection{SLM Layer 2: Abstract Resource Management}

This layer provides the ability to translate abstract performance objectives to raw resources requests, and to start execution and monitoring services on specified resources. However, in order to support these functionalities, the SLM needs to:

- Query resource providers for static resource characteristics and monitor dynamic characteristics of these resources.

- Acquire and manage resources (potentially across multiple providers). This implies maintaining aggregate resource views and keeping track of existing allocations.

- Submit and control job executions at these providers.

Heterogeneity is one significant practical obstacle at this layer: each resource manager at each provider may have its own protocols and APIs for the tasks enumerated above. If these resource managers are not Grid-enabled, this layer will need to gather information in a different way from each resource manager, translate this information to a uniform representation, aggregate it, and keep track of current allocations. Grid-enabled resource managers provide uniform, higher-level functionality (like aggregate views over managed resources and advanced monitoring mechanisms) that simplifies this SLM layer.

We note that, while the translation, arbitration, and allocation/aggregation functions presented above are implemented by the SLM as a whole, each of the SLM layers implement some, if not all, of these functions themselves. Thus, the behavior of the whole SLM is obtained by composing the functionalities of its layers. For example, performance objective translation is realized at both layer 1 and 2. Layer 1 uses an application specific performance model to translate application-level performance objectives into abstract resource objectives, while layer 2 uses resource status and configuration information to further translate these objectives into request for specific resources. Similarly, an aggregation function may be 
present at both these layers although with different goals: the higher layer might aggregate across resource providers (belonging to different administrative domains) while the lower layer might simply aggregate resources from within the same administrative domain.

Likewise, an arbitration function can also be present at all three layers. Multiple client requests for the same service may be arbitrated at layer 1, with layer 2 merely translating request objectives. Arbitration can also be pushed down to layer 2, if no aggregation is required at layer 1. A similar scenario is possible between layer 2 and layer 3 .

We envisage an architecture where these layers are loosely coupled and use SLAs to communicate performance objectives, utilities associated with requests, and monitoring and fault information from one layer to the next. We believe that, as these ideas gather acceptance and as standardization work matures, these mechanisms will be incorporated in the default resource management schemes deployed by the resource providers themselves in the same way schedulers with sophisticated functionality are adopted in today's cluster resource managers.

\section{Illustrating SLM Functions in Different Service and Resource Environments}

This section illustrates the use of three key SLM functions, i.e., translation, arbitration and allocation/aggregation, in actual SLM systems for various scenarios and design approaches. To underline our main points, we have chosen three concrete example systems, that are somewhat newer systems (i.e., recent prototypes) addressing less familiar scenarios, rather than illustrating these functions using well known examples of SLM systems, such as workload managers. Note that all three SLM functions are used in the design of an workload manager that classifies and prioritizes execution of incoming service requests, and then routes each request to a dynamically selected node for meeting average response time objectives for the requests coming from a specific user or user group, while balancing load across the server nodes. A service requester sees only the service abstraction and is not aware of the detailed implementation of this service. The translation function identifies the application instances to be used for servicing a request; the arbitration function prioritizes execution of incoming requests from multiple requesters; and the allocation function routes the request to a specific instance.

\subsection{SLA-based Parallel Application Service Management}

Our prototype system [43] manages parallel application service instances based on user defined SLAs. It translates end-user SLA requirements into concrete resource requirements that are then used to configure a parallel service instance based on a prior application execution profile. The system also schedules incoming user requests to match available preconfigured service instances, and proactively manages a pool of preconfigured service instances. 
We address the following scenario. Setting up a parallel service instance with the required resource configuration involves acquiring resources, starting up a (parallel) application on acquired nodes, and, after execution, shutting down the application and releasing resources. To avoid the overheads associated with per-request resource acquisition, an application service instance can be reused for serving multiple user requests with similar requirements.

However, the end-user need not specify exactly what resource configurations are needed for a particular request, and just specifies a high-level SLA which includes two metrics: the desired execution time for the request (translated in turn into the resource configuration requirements) and the wait time to acquire a matching service instance.

The architectural components that manage these metrics are illustrated in Figure 3. The two key components are the Application Service Level Manager (ASLM) and the Service Instance Pool Manager (SIPM). The ASLM maintains profiles for supported applications and extrapolates from known data-points to derive the exact resource configuration for meeting the first SLA objective: the user-specified execution time. Thus the ASLM translates a high-level request into a concrete request for a particular parallel application service instance. Once the request is translated to a particular resource configuration, the local scheduler binds the request to a service handle which is the preconfigured parallel application instance.

The SIPM allocates/de-allocates resources across the different service instance pools, so that the second metric, the wait time to obtain a service handle, is met. SIPM also arbitrates between service pools when insufficient resources are available based on the user specified business-value or relative importance of the requests specified as part of the SLA.

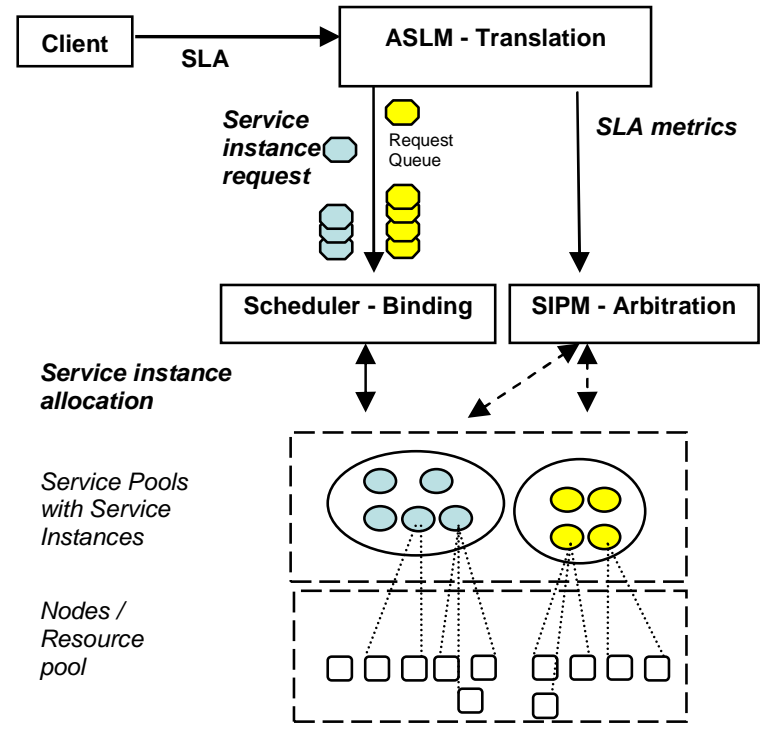

Figure 3: Architectural components for managing parallel application service instances 
While our main objective in this section is to present how the SLM architecture and design principles can be instantiated in a number of real-world scenarios we also bring evidence that these instantiations fully meet their requirements. The experiment described below, and in more detail in Ranganathan et al. [43], demonstrates that the SPIM allocates resources efficiently. The SIPM attempts to maximize a user-defined utility function: utility is defined with a linear dependency of service response time. As seen in Table 1, in the static allocation case where SPIM is not used, the average response time for service S1 is high, leading to low overall service utility. When the SIPM is used, the response times for S1 are effectively reduced whereas the response times for service S2 go up slightly resulting in positive revenues for both services. Note that the revenue numbers are entirely dependent on the utility function used, while the average response times are independent of whatever utility function is used.

These results, together with the exhaustive experimentation presented in Ranganathan et. al [43], show that SIPM is successful in adapting service instance pools, for maximizing utility, when compared to a static scheme where no adaptation across service pools occurs.

Table 1: Performance comparison of SIPM's dynamic re-provisioning to static provisioning: The request arrival rates for the two services S1 and S2 differ by a factor of two. Arrival times for requests for S1 follow a Poisson distribution, and arrive twice as often as requests for S2 (Poisson median inter-arrival time for S1 = 5, $\mathrm{S} 2=10)$. In the beginning of the simulation, each service pool contains 10 instances.

\begin{tabular}{|l|c|c|c|c|}
\hline & \multicolumn{2}{|c|}{ Avg. Response Time (secs) } & \multicolumn{2}{c|}{ Revenue (\$) } \\
\hline & Static & SIPM & Static & SIMP \\
\hline S1 & 578 & 31 & -2662 & 44 \\
\hline S2 & 2 & 14 & 95 & 63 \\
\hline Total revenue & $\mathrm{Na}$ & na & -2567 & 107 \\
\hline
\end{tabular}

\subsection{Model-based Translation: an Application Specific SLM Instantiation for Scientific Applications}

Cactus $[44,45]$ is a framework for developing scientific and engineering high-performance applications. Apart from its large user base, an additional reason to choose Cactus for prototyping a SLM was our extensive previous experience with this framework. The Cactus system can be deployed in heterogeneous distributed environments [46] and detailed performance models [24] have been developed.

Our goal with this prototype is to explore the feasibility of model-based translation, i.e., employ analytic equation-based performance models to derive resource requirements for Cactus based applications. Cactus system has been modified in order to expose these applications as Grid services [47] and the corresponding service factories implement the two SLM functionalities described for in Section 3.1. First, the service factory hides all application specific details of starting and configuring the computational framework. Second, the factory embeds an analytical, equation-based, performance model for the application and is able to translate high-level performance objectives (response time in this case) into lower level resource requirements (number of cluster nodes). To compute required resources, in 
addition to response time the analytical model takes into account application requirements that drive the memory usage: the size of the problem (i.e., the size of the physical space to be modeled) and simulation granularity. This model is described in detail in Ripeanu et al. [24].

With this prototype we deviate slightly from the architecture proposed in Section 3.1: once the translation to resource provider objectives is made, the service factory acquires resources from the resource provider. The factory then instantiates a new Cactus service and returns its handle for use. In addition to encapsulating application specific behavior in the service factory, this architecture brings additional performance gains by turning a traditional MPI application into a service. This way, application startup costs, which can be significant for a large MPI application, are amortized over multiple requests.

\subsection{GRUBER: Usage Policy-based Resource Management in Virtual Organizations}

In this section we explore a new direction where our layered architecture can be applied. GRUBER [48, 49] is a framework for resource management in Grid environments based on usage policies. Grids often aggregate hundreds from independent providers (e.g., a scientific community sharing resources deployed at participating institutions) and thousands of users. In these large deployments resource providers, e.g., organizations or individual users, aim to specify policies that control the use of the resources they offer to share: for example institutions might want to allocate a specific share of their resources to a specific project, virtual organization or individual user. While long time present in the networking domain [50, 51] for bandwidth allocation, resource management based on usage policies is new in the Grid space.

For reasons similar to those presented in Section 3, GRUBER enables providers to specify usage policies at a high level, namely at the abstract resource level. As a consequence, GRUBER's architectural layout is similar to that presented in Section 4.1 for the SLA-based service management for parallel applications. GRUBER implements Layers 1 and 2 of the design described in Section 3. It implements resource aggregation and arbitration among competing requests and a primitive form of translation. Aggregation is achieved by obtaining a view of all currently used and available resources (at various levels -- organization, groups, or user level). Arbitration functionality is implemented using priorities: together with resource usage policies, resource owners can specify usage priorities and associated time intervals at organization, site, and individual user level. These priorities are then translated to arbitrate between competing requests. By using monitoring and resource information obtained GRUBER, offers an interface at the abstract resource level (Section 2.2.2) and performs primitive translation requests for specific computational rates (e.g., expressed in MIPS or FLOPS) down to actual physical resources.

GRUBER'S architecture is composed of four principal components, as follows:

(a) Engine: Monitors and predicts resource availability and maintains a global view of resource utilization. Our implementation is a WSRF service [52] and benefits from 
additional authentication and authorization functionality provided by Globus Toolkit middleware.

(b) Site Monitor: This is the data provider for the GRUBER engine - it is composed of a set of UNIX and Globus tools for collecting grid status elements.

(c) Site Selectors: Use the data provided by the GRUBER engine to provide task assignment alternatives. Site selectors implement a number of task assignment policies, e.g., round robin, least used, or last recently used [53].

(d) The GRUBER client: Resides on the submitting host, monitors local organization' available resources, resource use and specified resource consumption policies and decides how many jobs to start and when.

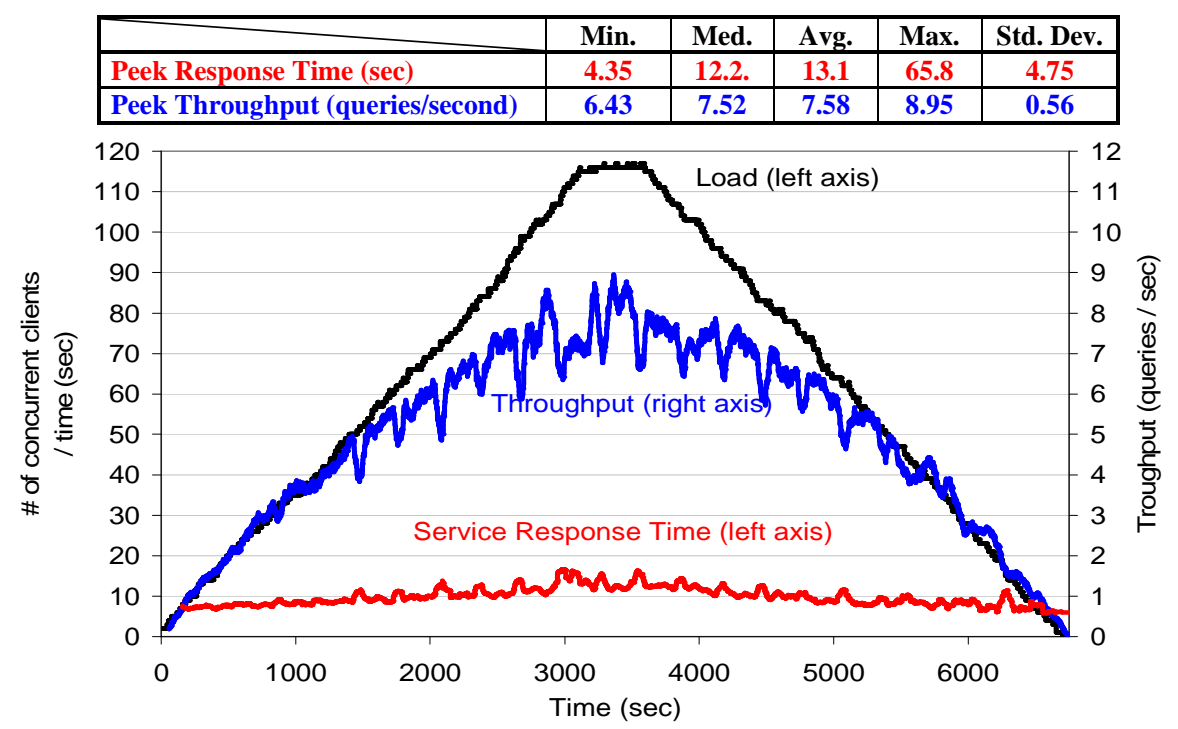

Figure 4: GRUBER's performance. We measure GRUBER performance using DiPerF [26]: load is generated incrementally by 115 wide-area clients controlled by DiPerF. Clients start one every 30s and submit a request when they start and immediately after they get a reply aiming to always have one outstanding request in the system. The average service response time is about $13 \mathrm{~s}$, and the achieved throughput reaches about 7.5 queries per second during the peak load period. We observe that GRUBER's throughput and response time remain relatively constant during peak offered load.

GRUBER's usage policies can be issued at multiple levels: virtual organization and site. Each individual user and institution may participate in and contribute resources to multiple collaborative projects that can vary widely in scale and lifetime. GRUBER employs usageSLAs to specify resource sharing policies and the usage policy enforcement problem involves deciding, at each submission point, which jobs to route to which sites and at what time, to both satisfy site and organization level usage-SLAs and, additionally, to optimize metrics such as resource utilization and overall job and workload execution times. We note that this model is one of resource sub-allocation: resources are owned by sites which apportion them 
to VOs. VOs in turn apportion their "virtual" resources to groups. Groups could, conceptually, apportion their sub-allocation further, among specific users.

Applications invoke site selectors to obtain a site recommendation, while the GRUBER queue manager is responsible for controlling job starting times. To manage a large number of resources distributed resources and large workloads in dynamic environments, GRUBER can be deployed as multiple coordinating decision points [48]. This architecture supports on-the-fly usage policy changes and bootstrapping of new virtual organizations. Figure 4 presents GRUBER performance when deployed as ten decision points and serving more than 110 geographically distributed clients: it demonstrates that GRUBER can scale to handle a large number of clients and offer the throughput and response time required by client applications.

\section{Composition of SLM Functions to Support New Scenarios}

SLM functions and existing SLM systems can be composed to address new scenarios. Section 5.1 presents general layering concepts while Section 5.2 describes how to mix two parts of the vertical stack in a concrete setting, using an orchestration layer.

\subsection{Layered Management Architecture for Managing Business Processes, Services, Transactions and Resources}

To further illustrate the concept of SLM composition and multiple layering in managing a complex system environment, consider Figure 5, where an end-client may request use of services or resources at various abstraction levels with corresponding service level objectives. An end-client may directly request the execution of a job or application, where the lowest layer, Job scheduling \& Resource allocation layer, arbitrates across multiple job execution requests, allocates resources to meet service level objectives both in terms resource objectives as well as delay in resource allocation, and finally, starts execution of the application on the allocated resources. Moving up the layering, an end-client submits a service request with the service level objectives expressed using average response time and/or throughput metrics. Each service request may be executed on a single node for a short interactive transaction or may be executed using a parallel application instance deployed over multiple nodes. As illustrated earlier, the two scenarios are managed differently either as workload management (i.e., prioritizing and routing requests) or by binding service requests to a parallel application service instance configured to meet the requester specified service level objectives. Both the interactive transactional application and parallel application management system layer in turn request resources and instantiate application instances as required, using the job scheduling and resource allocation layer. An end-client may also requests invocation of a composite service with a service level objective expressed over completion time metrics. The composite service management layer arbitrates across multiple client requests, and prioritizes execution of individual service steps arising from different end-client requests. In a monolithic centralized environment, without such a layering, the composite service management layer 
will make all the decisions, including when and how much resource to allocate to each composite service so as to meet the completion time objectives.

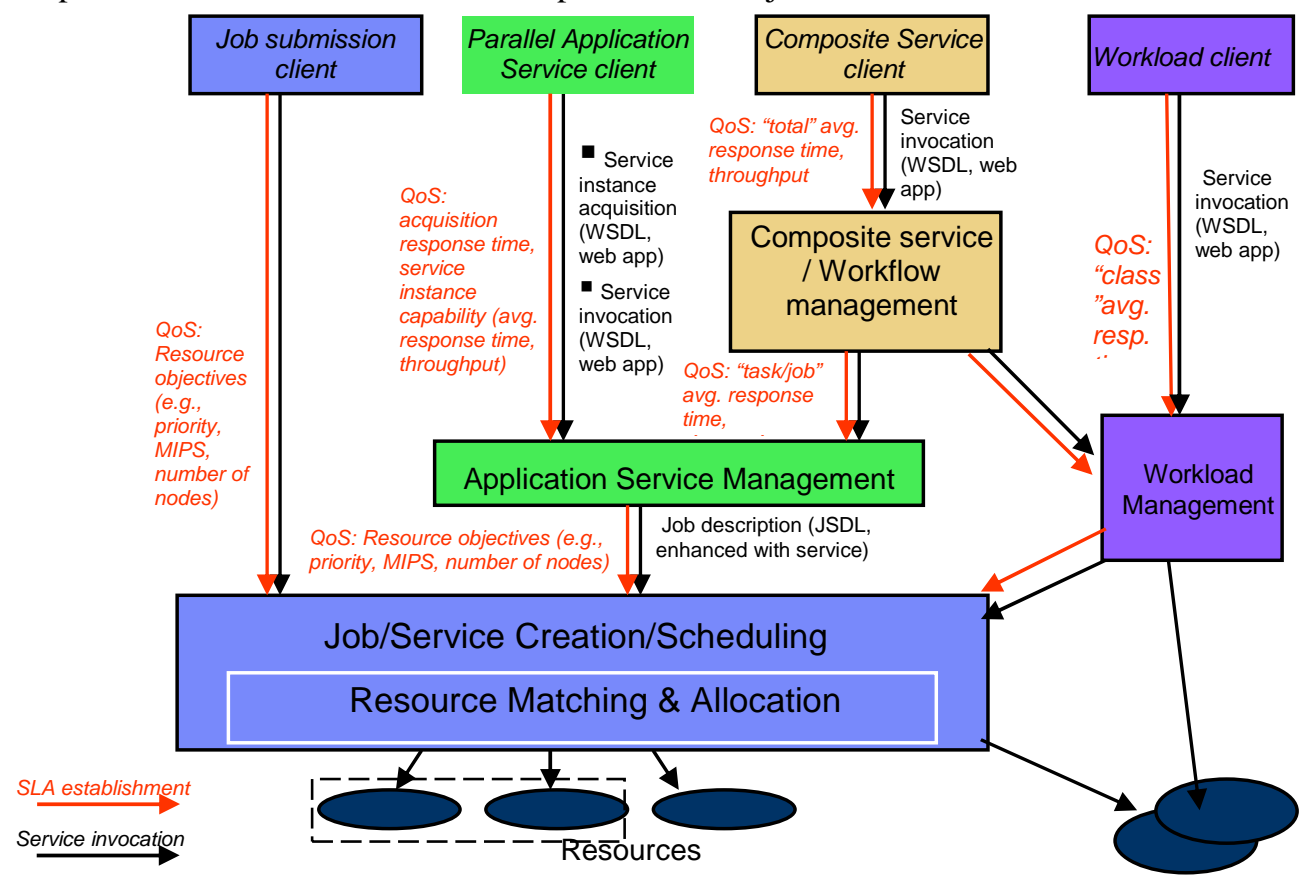

Figure 5: Layered management architecture for managing business processes, services, transactions and resources

\subsection{Managing Parallel and Interactive Applications}

As explained in section 4.1, parallel application service instances can be managed using high-level SLAs. To recall, the SIPM acts as an arbitrator and configures service instances to meet the client specified SLA metrics, for example the expected wait time to receive a handle to a service instance. However, other classes of applications need not fit this architecture for meeting client SLAs. For example, interactive applications traditionally use a workload manager which decides which particular server to forward requests to. There is however, a need for using a common resource pool for different modes of applications [54] in order to use resources more efficiently. Figure 6 outlines this scenario: the left side illustrates the parallel service instance mode of operation described in Section 4.1 while the right side denotes a typical interactive mode. The important observation is that both modes draw resources from a shared resource pool, according to current demand for their services.

However this scenario introduces a new problem: deciding how the resources in the pool are shared between the two operational modes. One architectural solution involves two SLA levels: one between the client and a high-level arbitrator like the SIPM, and a second SLA level between the SIPM and a common resource level arbitrator. Thus the arbitrator of each 
mode, the SIPM and the Workload Manager respectively, create new SLAs with the resource provider. In case of contention for resources between the two modes the resource provider now has to decide which SLA is more important. The global policy project [55] employs this architecture where high-level arbitrators specify POBs (probability of breach) which are then used by the low-level arbitrator to make decisions. A second, simpler architectural solution is to allow the SIPM and Workload Manager to explicitly request resources from the provider and provide an associated importance for their request, for example a business value associated with each request. The resource provider can then allocate resources solely based on these business values.

Another aspect to note is that clients can request for exclusive reservations to meet critical objectives. Separate SLAs for enabling advanced reservation of resources for important jobs, may make it more viable to share resources between parallel and interactive workflows.

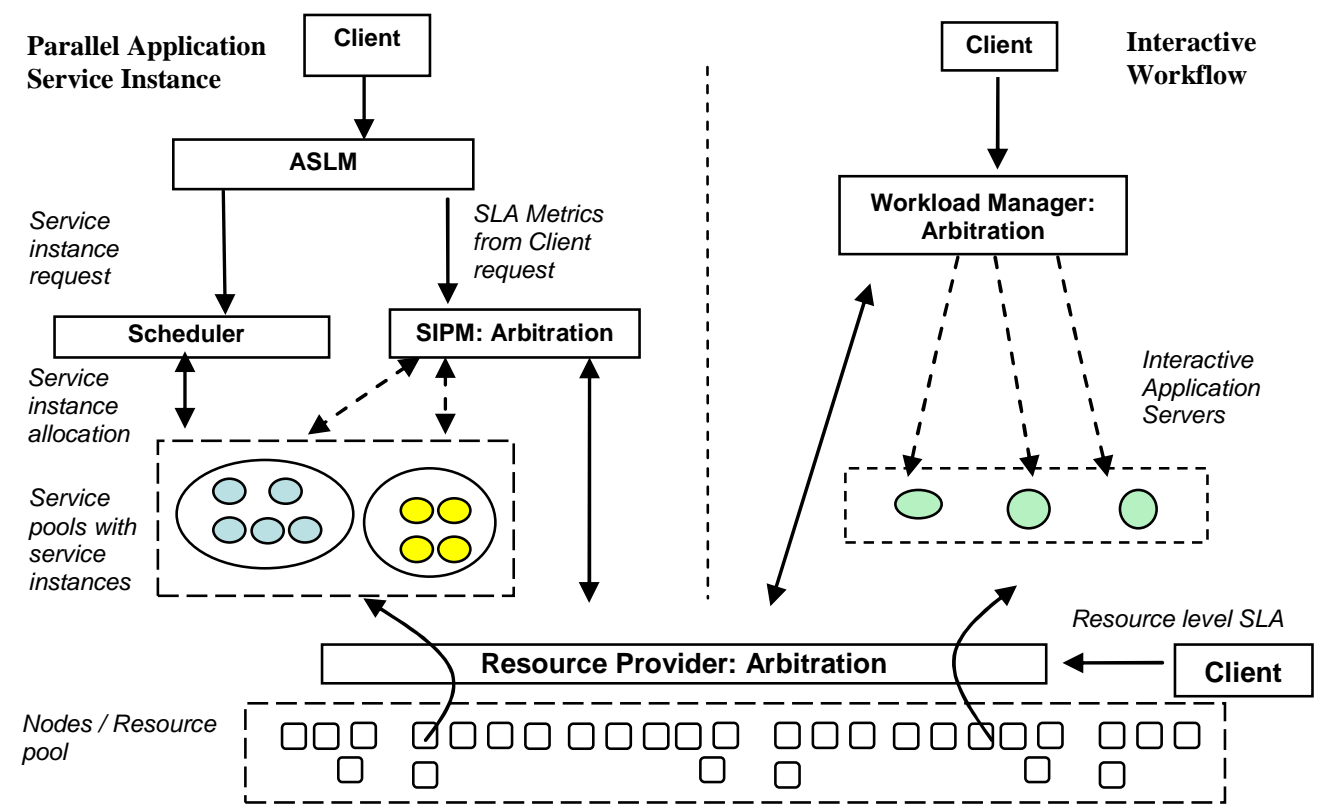

Figure 6: Architecture for managing parallel and interactive applications using a common pool of resources. Two levels of arbitration make this dual mode of operation possible.

\section{Summary}

This paper addresses the gap between the high-level specification of client resource requirements to meet specific performance objectives, and detailed specification of resources managed by resource providers. In the absence of a framework to bridge this gap, clients are forced to specify detailed required resource configurations in support of their performance objectives. We identify three levels of abstraction for specifying resource requirements a 
service provider needs to manage; namely: detailed specification of raw resources, virtualization of heterogeneous resources as abstract resources, and service level requirements at an application level. We also identify three key functions to manage service level agreements, namely: translation of resource requirements across virtualization layers, aggregation of resources from multiple lower level resource managers, and arbitration in allocating resources across client requests in managing service level agreements. We use a real world example from the financial industry, to illustrate the three abstraction layers and the use of these key SLM functions in managing these layers. Finally, we describe three prototypes that exploit the design principles presented to bridge the abstraction gap between high-level client requests and lower-level resource management objectives. These prototypes employ the layered architecture we advocate and employ, to various degrees, basic translation, aggregation and arbitration primitives.

\section{Acknowledgements}

We would like to thank Marcos Novaes for providing details on the example scenario and Ian Foster for his comments on the paper.

Part of this work was carried out while the Catalin Dumitrescu and Matei Ripeanu were graduate students with the Distributed Systems Laboratory, at the University of Chicago.

\section{References}

[1] I. Foster and C. Kesselman, The Grid: Blueprint for a New Computing Infrastructure (Second Edition): Morgan-Kaufmann, 2004.

[2] A. Andrieux, K. Czajkowski, A. Dan, K. Keahey, J. Pruyne, J. Rofrano, S. Tuecke, and M. Xu, Web Services Agreement Specification, Version 1.1, Draft 20, in Global Grid Forum, 2004.

[3] H. Ludwig, A. Dan, and R. Kearney, Cremona: An Architecture and Library for Creation and Monitoring of WS-Agreements, 2nd International Conference on Service Oriented Computing (ICSOC), 2004.

[4] D. Lamanna, J. Skene, and W. Emmerich, SLAng: A Language for Defining Service Level Agreements, 9th IEEE Workshop on Future Trends of Distributed Computing Systems, 2003.

[5] H. Ludwig, A. Keller, A. Dan, and R. King, A Service Level Agreement Language for Dynamic Electronic Services, 4th IEEE International Workshop on Advanced Issues of E-Commerce and Web-based Information Systems (WECWIS'02), Newport Beach, California, USA, 2002.

[6] WSLA Language Specification, Version 1.0, IBM Corporation, 2003.

[7] M. P. Papazoglou and D. Georgakopoulos, Service-oriented computing, Communications of the ACM, vol. 46, pp. 24-28, 2003.

[8] M. Novaes, Personal Communication, 2002.

[9] A. Leff, J. T. Rayfield, and D. M. Dias, Service-Level Agreements and Commercial Grids, IEEE Internet Computing, vol. 7, pp. 44-50, 2003.

[10] IBM Load Leveler: User's Guide, September 1993.

[11] The Maui Scheduler, http://www.supercluster.org/maui/. Center for HPC Cluster Resource Management and Scheduling, 2004.

[12] Platform Computing, Load Sharing Facility, http://www.platform.com, 2004. 
[13] K. Czajkowski, I. Foster, N. Karonis, C. Kesselman, S. Martin, W. Smith, and S. Tuecke, A Resource Management Architecture for Metacomputing Systems, in 4th Workshop on Job Scheduling Strategies for Parallel Processing: Springer-Verlag, 1998, pp. 62-82.

[14] A. Anjomshoaa, F. Brisard, R. L. Cook, D. K. Fellows, A. Ly, S. McGough, and D. Pulsipher, Job Submission Description Language (JSDL) Specification v0.3, Global Grid Forum 2004.

[15] J. J. Dongarra, J. DuCroz, I. Duff, and S. Hammerling, A Set of Level 3 Basic Linear Algebra Subprograms, ACM Transactions on Mathematical Software, vol. 16, pp. 1-17, 1990.

[16] A. Petitet, S. Blackford, J. Dongarra, B. Ellis, Graham Fagg, K. Roche, and S. Vadhiyar, Numerical Libraries And The Grid: The GrADS Experiments With ScaLAPACK, International Journal of High Performance Computing Applications, vol. 15, 2001.

[17] H. Casanova and J. Dongarra, NetSolve: A Network Server for Solving Computational Science Problems, International Journal of Supercomputer Applications and High Performance Computing, vol. 11, pp. 212-223, 1997.

[18] M. Vanneschi, The programming model of ASSIST, an environment for parallel and distributed portable applications, Parallel Computing, vol. 28, pp. 1709-1732, 2002.

[19] F. Berman, A. Chien, K. Cooper, J. Dongarra, I. Foster, D. Gannon, L. Johnsson, K. Kennedy, C. Kesselman, J. Mellor-Crummey, D. Reed, L. Torczon, and R. Wolski, The GrADS Project: Software Support for High-Level Grid Application Development, International Journal of High Performance Computing Applications, vol. 15, pp. 327-344, 2001.

[20] S. Gorlatch and J. Dünnweber, From Grid Middleware to Grid Applications: Bridging the Gap with HOCs, Future Generation Grids, 2005.

[21] G. Edjlali, G. Agrawal, A. Sussman, J. Humphries, and J. Saltz, Runtime and Compiler Support for Programming in Adaptive Parallel Environments, Scientiffic Programming, vol. 4, 1996.

[22] B. Urgaonkar, G. Pacifici, P. Shenoy, M. Spreitzer, A. Tantawi, An Analytical Model for Multitier Internet Services and Its Applications, ACM Sigmetrics Conference, Banff, Canada, 2005.

[23] R. Doyle, J. Chase, O. Asad, W. Jin, and A. Vahdat, Model-Based Resource Provisioning in a Web Service Utility, 4th USENIX Symposium on Internet Technologies and Systems (USITS), 2003.

[24] M. Ripeanu, A. Iamnitchi, and I. Foster, Cactus Application: Performance Predictions in Grid Environments, European Conference on Parallel Computing (EuroPar), 2001.

[25] S. B. Calo and D. Verma, Service Level Driven Provisioning of Outsourced IT Systems, IBM Systems Journal, 5(10), 2002.

[26] C. Dumitrescu, I. Raicu, M. Ripeanu, I. Foster, DiPerF: automated DIstributed PERformance testing Framework, 5th IEEE/ACM International Workshop on Grid Computing, 2004.

[27] G. Wrzesinskan, R. v. Nieuwpoort, J. Maassen, and H. E. Bal, Fault-tolerance, Malleability and Migration for Divide-and-Conquer Applications on the Grid, IPDPS 2005, Denver, CO, 2005.

[28] E. J. Posnak, R. G. Lavender, and H. M. Vin, An adaptive framework for developing multimedia software components, Communications of the ACM, vol. 40, pp. 43-47, 1997.

[29] R. Raman, M. Livny, and M. Solomon, Matchmaking: Distributed Resource Management for High Throughput Computing, IEEE International Symposium on High Performance Distributed Computing, 1998.

[30] C. Liu, L. Yang, I. Foster, and D. Angulo, Design and Evaluation of a Resource Selection Framework for Grid Applications, 11th IEEE International Symposium on High Performance Distributed Computing (HPDC-11), Edinburgh, Scotland, 2002.

[31] H. Tangmunarunkit, S. Decker, and C. Kesselman, Ontology-Based Resource Matching in the Grid - The Grid Meets the Semantic Web, International Semantic Web Conference, 2003.

[32] D. Oppenheimer, J. Albrecht, D. Patterson, and A. Vahdat, Design and Implementation Tradeoffs for Wide-Area Resource Discovery, 14th IEEE Symposium on High Performance Distributed Computing (HPDC), 2005.

[33] M. Litzkow, M. Livny, and M. Mutka, Condor - A Hunter of Idle Workstations, in Proc. 8th Intl Conf. on Distributed Computing Systems, 1988, pp. 104-111. 
[34] R. Henderson and D. Tweten, Portable Batch System: External Reference Specification, 1996.

[35] R. Wies, Using a classification of management policies for policy specification and policy transformation, Integrated Network Management, 1995.

[36] J. In, P. Avery, R. Cavanaugh, and S. Ranka, Policy Based Scheduling for Simple Quality of Service in Grid Computing, International Parallel \& Distributed Processing Symposium (IPDPS), Santa Fe, NM, 2004.

[37] D. Irwin, L. Grit, and J. Chase, Balancing Risk and Reward in Market-based Task Scheduling, HPDC-13, Honolulu, Hawaii, 2004.

[38] M. Balazinska, H. Balakrishnan, and M. Stonebraker, Contract-Based Load Management in Federated Distributed Systems, NSDI'04, San Francisco, CA, 2004.

[39] J. Aman, C. K. Eilert, D. Emmes, P. Yocom, and D. Dillenberger, Adaptive algorithms for managing a distributed data processing workload, IBM Systems Journal, vol. 36, 1997.

[40] A. Dan, D. Davis, R. Kearney, A. Keller, R. King, D. Kuebler, H. Ludwig, M. Polan, M. Spreitzer, and A. Youssef, Web services on demand: WSLA-driven automated management, IBM Systems Journal, vol. 43, pp. 136, 2004.

[41] I. Foster, C. Kesselman, C. Lee, R. Lindell, K. Nahrstedt, and A. Roy, A Distributed Resource Management Architecture that Supports Advance Reservations and Co-Allocation, Proc. International Workshop on Quality of Service, 1999.

[42] C. H. Crawford and A. Dan, eModel: Addressing the Need for a Flexible Modeling Framework in Autonomic Computing, MASCOTS, 2002.

[43] K. Ranganathan and A. Dan, Proactive Management of Service Instance Pools for meeting Service Level Objectives, 3rd International Conference on Service Oriented Computing (ICSOC), Amsterdam, Nederland, 2005.

[44] G. Allen, T. Goodale, G. Lanfermann, E. Seidel, W. Benger, H.-C. Hege, A. Merzky, J. Masso, T. Radke, J. Shalf, Solving Einstein's Equation on Supercomputers, IEEE Computer, pp. 52-59, 1999.

[45] W. Benger, I. Foster, J. Novotny, E. Seidel, J. Shalf, W. Smith, and P. Walker, Numerical Relativity in a Distributed Environment, Proc. 9th SIAM Conference on Parallel Processing for Scientific Computing, 1999.

[46] G. Allen, T. Dramlitsch, I. Foster, T. Goodale, N. Karonis, M. Ripeanu, E. Seidel, and B. Toonen, Supporting Efficient Execution in Heterogeneous Distributed Computing Environments with Cactus and Globus, SC'2001, Denver Colorado, 2001.

[47] S. Tuecke, K. Czajkowski, I. Foster, J. Frey, S. Graham, C. Kesselman, T. Maquire, T. Standholm, D. Snelling, and P. Vanderbilt, Open Grid Services Infrastructure (OGSI) version 1.0, Global Grid Forum, 2003.

[48] C. Dumitrescu, I. Raicu, and I. T. Foster, DI-GRUBER: A Distributed Approach for Grid Resource Brokering, SuperComputing, Seattle, WA, 2005.

[49] C. Dumitrescu and I. Foster, GRUBER: A Grid Resource Usage SLA Broker, 11th International Euro-Par Conference, Lisabona, Portugal, 2005.

[50] D. Verma, Simplifying Network Administration using Policy based Management, IEEE Network Magazine, 2002.

[51] J. Jin and K. Nahrstedt, QoS Specification Languages for Distributed Multimedia Applications: A Survey and Taxonomy, IEEE Multimedia Magazine, vol. 11, pp. 74-87, 2004.

[52] Web Services Resource Framework: http://www.globus.org/wsrf/, 2004.

[53] C. Dumitrescu, M. Wilde, and I. Foster, Model for Usage Policy-based Resource Allocation in Grids, 6th IEEE International Workshop on Policies for Distributed Systems and Networks (POLICY 2005), Stockholm, Sweden, 2005.

[54] Ochestration for On Demand Data Centers, IBM Research White paper, 2005.

[55] D. Rosu and A. Dan, Managing End-to-End Lifecycle of Global Service, 3rd International Conference on Service Oriented Computing (ICSOC), Amsterdam, Nederland, 2005. 